\title{
Alterstice
}

Revue internationale de la recherche interculturelle

International Journal of Intercultural Research

Revista International de la Investigacion Intercultural

\section{De la plainte aux soins : le parcours des victimes de violence conjugale au Brésil}

\author{
Lucienne Martins-Borges, Daniela Sevegnani Mayorca et Mariana Silva \\ Livramento
}

Volume 3, numéro 2, 2013

Violence conjugale et diversité culturelle

URI : https://id.erudit.org/iderudit/1077518ar

DOI : https://doi.org/10.7202/1077518ar

Aller au sommaire du numéro

\section{Éditeur(s)}

Alterstice

ISSN

1923-919X (numérique)

Découvrir la revue

Citer cet article

Martins-Borges, L., Sevegnani Mayorca, D. \& Silva Livramento, M. (2013). De la plainte aux soins : le parcours des victimes de violence conjugale au Brésil. Alterstice, 3(2), 27-35. https://doi.org/10.7202/1077518ar
Résumé de l'article

En Amérique latine, la violence conjugale touche $25 \%$ à $50 \%$ des femmes. Selon la Société mondiale de victimologie, $23 \%$ des Brésiliennes ont déjà été victimes de violence conjugale et, dans $70 \%$ des cas, il s'agissait de violence physique. Les protocoles d'intervention dans le cas de violence conjugale au Brésil varient d'une région à une autre mais impliquent, généralement, la participation du corps policier. Nous présentons ici le parcours des femmes victimes de violence conjugale lorsqu'elles décident de porter plainte à la police, et ce, jusqu'à la demande en soins psychologiques comme moyen de sortir de cette situation de violence. Les observations sont issues d'une étude auprès d'intervenants en institutions ayant pour mandat d'assister les femmes victimes de violence conjugale ainsi que d'un projet d'intervention psychologique pour personnes victimes directes ou indirectes de violence conjugale. Les activités de ce programme sont réalisées à la clinique de psychologie (Serviço de Atenção Psicológica) du Département de psychologie de l'Université Fédérale de Santa Catarina (UFSC). Elles ont pour objectif d'aider ces femmes à se réapproprier des éléments de leur histoire personnelle détruits par leur vécu de violence et à revaloriser leur identité au-delà de leurs rôles d'épouse et de mère. Nous avons également identifié certaines particularités culturelles ayant aidé ces femmes à faire face à leur situation, particularités représentant aussi parfois des limites et susceptibles de bloquer leur processus de changement. (c) Lucienne Martins-Borges, Daniela Sevegnani Mayorca et Mariana Silva Livramento, 2013
Ce document est protégé par la loi sur le droit d'auteur. L’utilisation des services d’Érudit (y compris la reproduction) est assujettie à sa politique d'utilisation que vous pouvez consulter en ligne.

https://apropos.erudit.org/fr/usagers/politique-dutilisation/ 


\title{
구
}

alterstice

Revue Internationale

de la Recherche Interculturelle

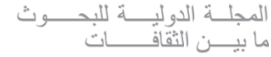

Revista International

de la Investigacion Intercultural

Revista Internacional de Pesquisa Intercultural

International Journal of Intercultural Research

ARTICLE THÉMATIQUE

\section{De la plainte aux soins : le parcours des victimes de violence conjugale au Brésil}

\author{
Lucienne Martins-Borges ${ }^{1}$, Daniela Sevegnani Mayorca ${ }^{1}$ et Mariana Silva Livramento ${ }^{2}$
}

\section{Résumé}

En Amérique latine, la violence conjugale touche $25 \%$ à $50 \%$ des femmes. Selon la Société mondiale de victimologie, $23 \%$ des Brésiliennes ont déjà été victimes de violence conjugale et, dans $70 \%$ des cas, il s'agissait de violence physique. Les protocoles d'intervention dans le cas de violence conjugale au Brésil varient d'une région à une autre mais impliquent, généralement, la participation du corps policier. Nous présentons ici le parcours des femmes victimes de violence conjugale lorsqu'elles décident de porter plainte à la police, et ce, jusqu'à la demande en soins psychologiques comme moyen de sortir de cette situation de violence. Les observations sont issues d'une étude auprès d'intervenants en institutions ayant pour mandat d'assister les femmes victimes de violence conjugale ainsi que d'un projet d'intervention psychologique pour personnes victimes directes ou indirectes de violence conjugale. Les activités de ce programme sont réalisées à la clinique de psychologie (Serviço de Atenção Psicológica) du Département de psychologie de l'Université Fédérale de Santa Catarina (UFSC). Elles ont pour objectif d'aider ces femmes à se réapproprier des éléments de leur histoire personnelle détruits par leur vécu de violence et à revaloriser leur identité au-delà de leurs rôles d'épouse et de mère. Nous avons également identifié certaines particularités culturelles ayant aidé ces femmes à faire face à leur situation, particularités représentant aussi parfois des limites et susceptibles de bloquer leur processus de changement.

\section{Rattachement des auteures}

${ }^{1}$ Universidade Federal de Santa Catarina, Brésil; ${ }^{2}$ Fundação Catarinence de Educação Especial - FCEE, Brésil.

\section{Correspondance}

lucienne.borges@ufsc.br

\section{Mots clés}

violence conjugale; cothérapie interculturelle; psychologie clinique interculturelle

\section{Pour citer cet article :}

Martins-Borges, L., Sevegnani Mayorca, D. et Silva Livramento, M. (2013). De la plainte aux soins : le parcours des victimes de violence conjugale au Brésil. Alterstice, 3(2), 27-35. 


\section{Introduction}

La violence se présente sous diverses formes dans les dynamiques des relations humaines. Parmi les variantes de violence, on retrouve la violence conjugale et l'homicide des femmes. Plusieurs auteurs, sans forcément placer I'homicide conjugal dans la continuité de la violence conjugale, attirent l'attention sur la proximité entre ces deux thématiques (Campbell, Webster et Glass, 2009; Martins-Borges et Leveillée, 2005; Wilson et Daly, 1993). En ce qui a trait à cette catégorie d'homicides - c'est-à-dire l'homicide des femmes -, le Brésil se place au deuxième rang en Amérique latine et au septième rang parmi les 84 pays pour lequels des données ont été recueillies par l'Organisation mondiale de la santé pour les années 2006 à 2010 (Waiselfisz, 2012). Rappelons que le Brésil est le plus grand pays en superficie et en population de l'Amérique latine, avec environ 195 millions d'habitants.

Le Sistema de Informação de Agravos de Notificação (SINAN) du ministère de la Santé du Brésil a pour mandat de compiler les interventions faites dans les institutions du réseau public au cours desquelles il est constaté que des enfants, des adolescents, des femmes ou des personnes âgées ont été exposés à la violence conjugale ${ }^{1}$, à la violence sexuelle ou à d'autres types de violence (pas seulement familiale). Sur les 70285 notifications enregistrées en $2011,65,4 \%$ concernaient des femmes (Waiselfisz, 2012). La violence physique était la forme de violence la plus fréquemment signalée chez les femmes de plus de 15 ans, le conjoint ou l'ex-conjoint se révélant l'agresseur dans $43,4 \%$ des cas. Chez les femmes de 20 à 49 ans, $65 \%$ des agressions physiques avaient été commises par le conjoint ou l'ex-conjoint et, dans 51 \% des cas, plusieurs épisodes de violence avaient eu lieu. En deuxième place se plaçait la violence psychologique, suivie de la violence sexuelle (Waiselfisz, 2012).

Selon le ministère aux Politiques féminines (Secretaria de Políticas para as Mulheres, SPM), en 2011, le centre d'appel pour les femmes (Central de Atendimento à Mulher - Disque 180, CAM-D180) a reçu 667116 appels téléphoniques et, parmi ces appels, 74984 (environ $11 \%$ ) concernaient des plaintes pour violence. De ce nombre, $61,3 \%$ se référaient à des épisodes de violence physique, $24 \%$ de violence psychologique et $10,9 \%$ de violence verbale. Environ 91 \% des appels pour agression concernaient une agression commise par un conjoint ou un exconjoint de la victime et, dans $40,6 \%$ des cas, la durée du lien avec le partenaire était de plus de 10 ans (SPM, 2011). En ce qui a trait à l'âge des femmes ayant fait appel à ce service, plus de $70 \%$ avaient entre 20 et 49 ans. Dans $80,3 \%$ des cas, les gestes de violence étaient de fréquence élevée : ils arrivaient tous les jours ou toutes les semaines (SPM, 2011). Il est à noter que, parmi ces femmes, 59,5\% ne se disaient pas dépendantes financièrement de leur conjoint. De plus, selon les informations obtenues, 58,3\% des agressions n'avaient pas eu lieu sous l'effet de l'alcool ou de la drogue (SPM, 2011). Ces chiffres mettent en évidence l'ampleur du problème de la violence conjugale au Brésil et la nécessité de l'analyser pour mieux comprendre les dynamiques impliquées dans ce phénomène et, par conséquent, pour mieux intervenir pour l'éradiquer.

\section{Démarche méthodologique}

Les observations présentées ici sont issues de deux sources, constituant deux étapes dans la recherche. Premièrement, une étude qualitative a été réalisée auprès de 17 intervenants (travailleurs sociaux, conseillers juridiques et psychologues) ayant au moins cinq années d'expérience professionnelle et pratiquant au sein d'institutions publiques, avec le mandat d'assister les femmes victimes de violence conjugale ainsi que leurs enfants. Deuxièmement, un projet pilote d'intervention psychologique pour personnes en situation de violence conjugale a été lancé au Brésil - plus spécifiquement dans une ville du sud du pays d'environ 600000 habitants. Pour ce qui est de la première étape, la recherche s'est appuyée sur des entrevues semi-structurées, réalisées dans les locaux de travail des intervenants participants. Les entrevues avaient pour objectif principal d'identifier les démarches successives entreprises par les femmes lorsqu'elles se décidaient à parler de la violence conjugale qu'elles vivaient en vue d'obtenir aide et soutien.

\footnotetext{
${ }^{1}$ Au Brésil, le terme « violence domestique » est le plus répandu pour faire référence à cette problématique, que ce soit dans les textes scientifiques ou législatifs. Nous privilégierons cependant tout au long de ce travail l'expression « violence conjugale ».
} 
En ce qui concerne la seconde partie de la recherche, soit le projet d'intervention psychologique, celui-ci a été mis sur pied à la suite des conclusions de l'étude ci-dessus mentionnée, et ce d'autant plus qu'il n'existait aucune autre ressource spécialisée dans cette municipalité. Des interventions cliniques, réalisée selon le modèle de la cothérapie interculturelle (Pocreau et Martins-Borges, 2013), ont lieu depuis mars 2010 dans le cadre du projet pilote ouvert à la communauté, plus précisément à la clinique de psychologie du Département de psychologie de l'Université fédérale de Santa Catarina, au Brésil (SAPSI/UFSC). Les consultations sont conduites par des stagiaires/internes et par des professeurs du département. Le modèle de la cothérapie interculturelle considère la culture comme une façon particulière d'être en relation avec l'Autre, avec soi-même et avec ses savoirs. Cette approche initialement développée auprès de réfugiés se montre tout à fait pertinente dans l'intervention auprès de personnes ayant vécu d'autres formes de violence. Les interventions du thérapeute principal et du cothérapeute principal approfondissent trois dimensions : un travail sur les liens d'origine - liens d'appartenance et liens actuels, soit un travail sur les premiers attachements-, un travail sur les différentes dimensions identitaires en lien avec la singularité de l'Autre et un travail sur la cohérence et le sens des situations vécues, passées et actuelles.

Les personnes qui viennent consulter dans le cadre de ce projet sont envoyées par des professionnels des institutions de la santé et des services sociaux (centres de santé et des services sociaux, hôpitaux, etc.) ou par le corps policier, ou bien elles viennent par elles-mêmes. Jusqu'à présent, il s'est agi majoritairement de femmes adultes. Pour le propos de cet article, nous avons exrait les observations cliniques de 9 études de cas. Les femmes étaient âgées de 20 à 55 ans, avec une majorité ayant entre 31 et 35 ans (4); 6 femmes vivaient encore avec leur conjoint et étaient liées à lui par le mariage; 7 femmes travaillaient à l'extérieur de la maison et 5 d'entre elles avaient un diplôme d'études supérieures. Tout au long de la mise en place de ce projet d'intervention, l'accent a été mis sur l'identification des voies choisies par les femmes pour parler de la violence (ou et à qui), sur la compréhension des représentations de la violence - surtout conjugale - ainsi que sur ce qui les a conduit à rester dans des relations marquées par la violence.

Le terme conjugal est ici utilisé pour désigner toutes les relations au sein desquelles des personnes sont liées l'une à l'autre ou l'ont été par le mariage, l'union stable ou la fréquentation. L'objectif de cet article est de présenter des réflexions théoriques et cliniques susceptibles d'enrichir l'intervention dans des situations de violence conjugale au sein des contextes organisationnel, culturel et social brésiliens. Pour mieux comprendre le parcours de plainte pour violence conjugale dans ce contexte, nous présentons également les principales politiques publiques brésiliennes et les directives sur lesquelles s'appuient les organismes impliqués dans la lutte contre la violence conjugale.

\section{Observations : les voies de la plainte pour violence conjugale}

Il est important de noter que ces femmes, dans leur recherche de moyens pour mettre fin à la violence, ne cherchent pas nécessairement, du moins dans un premier temps, des moyens pour mettre fin à la relation. Cette demande est même rare chez les femmes rencontrées. Ce qu'elles expriment, généralement, c'est plutôt une demande d'aide pour que cesse la violence et que la relation puisse continuer. Mais comment et où font-elles cette demande ? Trois catégories d'aide permettent de décrire les parcours entrepris par les femmes qui fréquentaient les institutions où travaillaient les intervenants et participants à notre enquête: la famille et l'Église (de toutes confessions), la sécurité publique et la justice et enfin la santé et les services sociaux. Chacune de ces catégories est présentée ci-dessous, et ce, de la façon dont elle a été évoquée par les femmes participantes.

\section{La famille et l'Église}

En ce qui a trait à la recherche d'aide auprès des familles - la famille du conjoint et, par la suite, sa propre famille -, la femme tend à être confrontée à une alternative : comprendre son conjoint ou s'en séparer. En ce qui concerne la première possibilité, elle est incitée à poursuivre la relation et à comprendre ce qui arrive à son conjoint. Dans ce cas, la violence est souvent expliquée par les membres des familles - souvent la belle-mère, les belles-sœurs, la mère ou les sœurs, donc principalement des femmes - comme étant la conséquence d'une surcharge de problèmes portés par le conjoint (les préoccupations financières, le travail, le stress, etc.). L'environnement familial consulté est sensible à la souffrance de la femme, mais explique la violence du conjoint comme étant une réaction compréhensible dans les conditions de vie de de dernier. Les réactions de la femme sont alors questionnées (a-telle réagi de la manière attendue lorsque le conjoint s'est montré préoccupé, surchargé, fatigué, etc. ?), ceci afin de 
savoir si elle a pu contribuer d'une manière ou d'une autre à l'agression et ce qu'elle a fait (ou aurait dû faire) pour l'éviter. Dans le second cas, au contraire, l'acte du conjoint est blâmé, considéré comme inacceptable et il est fortement conseillé à la femme de quitter ce dernier, surtout si elle souhaite recevoir de l'aide de sa famille. En règle générale, les femmes ont souvent des difficultés à entendre cette possibilité et, lorsqu'elles reprennent la vie commune et que la violence se manifeste de nouveau, elles sont envahies par la honte d'avoir à redemander de l'aide à leur famille. Face à cette alternative - maintenir le lien et " comprendre la situation » ou se séparer de son conjoint -, les femmes se sentent de plus en plus coupables, seules et démunies après chaque nouvel épisode.

L'aide auprès des Églises (catholiques, protestantes, néoprotestantes, spirites, etc.) apparaît concomitamment aux demandes de la femme à sa famille (tous deux constituant des éléments essentiels du réseau affectif et social), et ce qu'elle entend va soit dans le sens de la compréhension et de la tolérance, soit dans le sens de sa part de responsabilité (qu'elle vit sur le mode de la culpabilité).

\section{La sécurité publique et la justice}

Après avoir eu recours à la famille, certaines femmes décident - souvent comme geste ultime - de faire appel à la police et à la justice. Leur demande va - à nouveau - dans le même sens : elles souhaitent de l'aide pour en finir avec la violence... mais pas avec le mariage ni avec la relation. Comment se passe cette demande d'aide auprès des représentants de la sécurité et de la justice? Les femmes arrivent envoyées par des membres de la famille (qui voient la séparation comme moyen de mettre un terme à la violence), par des membres du réseau social (autre que la famille) ou elles viennent d'elles-mêmes lorsqu'elles se voient en situation de danger. Cette démarche est vécue dans tous les cas par ces femmes comme une expérience de détresse et elles se voient impliquées dans des procédures légales sans avoir les moyens d'y faire face et de les contrôler. Elles ont affirmé s'être senties insécurisées et impuissantes face à la complexité de ces procédures et ont été envahies par la culpabilité - par rapport au conjoint mais également par rapport aux enfants. Cette voie de la plainte faisant appel à la sécurité et à la justice est effectivement complexe et nécessite certains éclaircissements pour être comprise correctement.

La Loi Maria da Penha 11.340/2006 précise les mécanismes légaux visant à empêcher la violence conjugale et familiale au Brésil. Cette loi a été nommée Loi Maria da Penha en hommage à une femme qui, en 1983 - après un passé de violence conjugale qui a abouti à une tentative d'homicide la laissant paraplégique -, a décidé de dénoncer cette violence à la justice brésilienne. Lors du procès, l'accusé (le conjoint) a été déclaré innocent. Cette décision de la Cour a incité Maria da Penha à recourir au Comité latino-américain pour la défense des droits de la femme (CLADEM) et au Centre pour la justice et le droit international (CEJIL), pour enfin s'adresser à la Convention américaine relative aux droits de l'homme de l'Organisation des États américains (CIDH-OEA). Cette démarche a eu pour conséquence la recommandation d'une réforme du système législatif brésilien pour la lutte contre la violence conjugale (Izumino, 2007). En 1984, cette recommandation, renforcée par la signature du gouvernement brésilien de la Convention pour l'élimination de toutes les formes de discrimination à l'égard des femmes (CEDAW-ONU), a eu pour effet d'inclure dans la Constitution fédérale brésilienne, entrée en vigueur à la fin de la dictature (1988), le texte légal sur le besoin de promouvoir l'égalité et l'équité entre les hommes et les femmes (Alves, 2006), et ce, dans tous les domaines. Maria da Penha est aujourd'hui l'une des figures de proue du mouvement de défense des droits des femmes au Brésil.

L'urgence et la nécessité de prendre en compte et de consolider les demandes des mouvements féministes ont été reconnues et des mesures institutionnelles ont été mises en place progressivement (élaboration de lois, création d'institutions et de services). En 1985, un service de police spécialisé pour les femmes (Delegacias Especializadas de Atendimento à Mulher, DEAM) a commencé à être implanté, avec l'objectif de répondre aux besoins des femmes en situation de violence conjugale. La DEAM de la municipalité concernée par la seconde partie de notre recherche a été la deuxième à avoir été créée au Brésil.

Une étude réalisée par le CAM-D180 sur l'impact de la Loi Maria da Penha a montré que, avant l'adoption de cette loi, 3356 appels étaient reçus en moyenne chaque mois. Après son adoption, ce nombre est passé à 6600 par mois, soit une augmentation de $96 \%$ de la demande pour ces services (SPM, 2012). 
Lorsque la femme arrive à la DEAM ou lorsqu'elle y est envoyée par des intervenants d'autres institutions publiques ou privées, elle y est accueillie par les intervenants de la DEAM elle-même qui, après avoir entendu sa plainte, l'informent de ses droits ainsi que des procédures légales découlant de cette plainte. Si elle décide de maintenir sa plainte, elle est dirigée vers un agent de police, qui remplit au registe du poste de police un bulletin d'événement (Boletim de Ocorrência, BO). En l'absence de blessures physiques, la femme peut déposer une plainte sans qu'un procès au criminel soit amorcé. Dans le cas de blessures physiques, l'agent dirige la plainte vers le ministère public (Ministério Público), indépendamment de la volonté de la plaignante, comme le prévoit le code civil brésilien (Código Civil Brasileiro). À partir du moment où les procédures ont été entamées par le ministère public, la femme doit subir un examen physique (médecin légiste), trouver des témoins et commencer éventuellement les procédures de séparation. Après l'émission du bulletin d'événement, le pouvoir judiciaire a 48 heures pour émettre la mesure protectrice qui définira la distance physique envers la plaignante et les membres de sa famille que l'accusé devra respecter jusqu'au jour de sa comparution. Lors de cette dernière, la femme, son conjoint et les témoins sont convoqués pour établir la véracité des faits et pour confirmer l'intention de donner suite au processus. Depuis le 9 février 2012, le Sénat brésilien a cependant approuvé une importante modification à la Loi Maria da Penha : il a été établi que, pour les dénonciations de violence pratiquée contre la femme sous forme de violence physique, avec lésions ou non, il n'est plus nécessaire d'attendre l'autorisation de la plaignante pour la poursuite de procédures judiciaires par le ministère public (Supremo Tribunal Federal, 2012).

Selon le chef de police de la DEAM de la municipalité concernée par notre recherche, environ $80 \%$ des femmes se désistent de la plainte qu'elles ont déposée, à des étapes différentes des procédures, parfois même après l'entame de démarches par le ministère public. Les limites financières, les menaces faites par le conjoint ou la reprise de la vie conjugale constituent les motivations les plus fréquemment invoquées lorsqu'elles décident de mettre fin aux procédures et aux mesures de protection instaurées par le ministère public.

Le Centre de référence pour femmes victimes (Centro de Referência à Mulher em Situação de Violência, CREMV) de cette municipalité, créé en 2009, relève du Bureau municipal d'assistance sociale (Secretaria Municipal de Assistência Social) et se situe à côté de la DEAM. Ce centre se définit comme un lieu d'accueil, d'intervention sociale, d'aide psychologique et d'orientation juridique (Prefeitura Municipal de Florianópolis, 2012) ayant comme objectif général de mettre fin à la situation de violence dans laquelle se trouvent les femmes. II reçoit les femmes envoyées par la DEAM et par d'autres institutions et constitue un lieu central dans l'organisation des services offerts aux femmes en situation de violence. Tout comme ceux de la DEAM, les professionnels de l'équipe du CREMV informent les femmes de leurs droits et de leurs devoirs et, si nécessaire, leur suggèrent d'entreprendre un suivi avec un professionnel de la santé ou des services sociaux.

\section{La santé et les services sociaux}

Les intervenants du réseau de la santé et des services sociaux offrent ainsi une troisième piste aux femmes pour parler de la violence qu'elles vivent. Cependant, ce choix intervient généralement après l'épuisement des deux autres modalités présentées ci-dessus (la famille et l’Église, la sécurité publique et la justice). Lorsque les femmes entrent en contact avec des intervenants de la sécurité publique et de la justice - comme nous l'avons décrit plus haut - elles sont dirigées soit vers des cliniques sociales (des cliniques en psychologie dont les tarifs sont bas), soit vers des centres de santé du réseau public pour un suivi en psychothérapie, soit encore vers des maisons d'hébergement pour femmes victimes de violence conjugale. Toutefois, surtout pour les femmes à faible revenu ou sans revenu, les possibilités de soin sont insuffisantes: elles ne pourront pas défrayer les coûts des cliniques sociales et les soins en psychologies offerts par le réseau public sont nettement insuffisants pour répondre à la demande. C'est à partir de ces constats que le projet pilote d'intervention psychologique pour personnes en situation de violence conjugale a été pensé et réalisé. Ce programme de suivi psychologique offert par l’Université s'inscrit donc dans la thématique de la violence faite aux femmes et représente une alternative pour celles qui décident de faire appel à la psychothérapie pour sortir de la dynamique de la violence. 


\section{Les femmes de la clinique et les " classiques de la violence conjugale »}

On observe chez les femmes reçues au programme d'intervention psychologique des façons similaires de s'exprimer, en thérapie comme dans la vie. Ces sont des modes d'expression qui, malgré leur histoire individuelle et donc singulière, correspondent à ce qu'on appelle les « classiques de la violence conjugale ».

En premier lieu, même si ces femmes sont originaires de milieux socioéconomiques variés (sans que leur appartenance soit donc limitée aux groupes socioéconomiques les plus vulnérables), elles finissent en général par abandonner leurs études et leur profession pour assumer, presque exclusivement, des fonctions de soin : soin des enfants, soin du conjoint, soin de la maison. Fréquemment, après leur mariage, elles migrent vers des villes ou des quartiers (dans le cas de grandes villes) éloignés de leur famille d'origine et de leurs amis. Par la suite, leurs tentatives de rompre avec cet isolement sont souvent blâmées, avec hostilité, par leur conjoint. Cela a pour conséquence de les enfermer dans un espace privé et restreint, ce qui fragilise leur réseau social et réduit leurs possibilités de choix et les empêche de sortir de la relation violente et de reprendre le contrôle de leur vie.

Ces femmes présentent également des difficultés à établir des limites claires entre soi et les autres lorsqu'elles expriment des désirs et des opinions et lorsqu'elles parlent de leurs projets de vie et de leurs façons d'être. Elles tendent à s'exprimer par le biais de la voix d'un autre qui, selon elles, est plus compétent pour parler des choses de la vie, voire d'elles-mêmes. Elles se montrent, au début des consultations (ce qui peut durer des mois), incapables de parler en leur nom, cette difficulté étant partiellement le résultat des nombreuses fois où leurs souhaits, leurs idées et leurs points de vue ont été invalidés. Ce renoncement à soi, voire cette inhibition du soi, a des conséquences sur leur subjectivité et sur leur capacité d'élaboration, ce qui rend difficile le travail clinique touchant la restauration narcissique et le renforcement de l'identité.

Dans une deuxième catégorie d'observations, nous avons relevé qu'il est fréquent de voir que ces femmes suivies en thérapie ont comme caractéristique, dans le setting thérapeutique, d'entamer ce suivi avec l'objectif d'aider les autres - généralement leur conjoint et leurs enfants. Elles trouvent une place en thérapie en passant par la préoccupation à l'égard des membres de leur famille. Ce fait dénote deux choses : que la violence fait souffrir la personne directement visée mais également les autres membres du noyau familial et que la femme considère que sa souffrance doit être entendue car elle rapporte la souffrance des autres membres impliqués et lui cherche des solutions. Elles ont difficultés à parler d'elles-mêmes en utilisant le "je » et elles ont recours à des termes génériques tels que le " tu », " les personnes ", " les femmes » comme sujets grammaticaux de leur récit. Le « je » comme sujet principal prendra du temps à émerger dans le discours. Cette difficulté, observée chez la majorité des patients en début de processus psychothérapeutique, a une connotation particulière dans le contexte de la violence conjugale, car il peut être considéré comme l'expression de l'affaiblissement du soi. Mariz (2008) soulève que cet engourdissement de la capacité de penser et de parler pour soi-même peut s'expliquer par la distance prise par la conscience comme moyen de défense contre la douleur psychique ressentie lors des épisodes d’agression.

Concernant la troisième catégorie d'observations - la famille des femmes en situation de violence conjugale -, il est remarquable de constater combien est forte la ressemblance entre leur père et leur conjoint. Les deux sont décrits comme contrôlants et agressifs. Dans leurs discours, il arrive qu'il soit difficile de savoir si elles parlent de la relation à leur père ou de celle à leur conjoint, ce qui traduit une confusion, qui semble intériorisée, au sujet des places respectives de chacun. Il est fréquent qu'elles aient quitté la maison familiale très tôt, encore adolescentes, soit parce qu'elles étaient enceintes, soit pour se marier. Dans ces conditions, on peut penser que leur contexte familial était insatisfaisant et qu'elles avaient l'espoir de trouver ailleurs et dans une autre relation une proximité affective absente dans leur famille d'origine. On observe également que leur conjoint comme leur père faisaient un usage abusif d'alcool et que leur agressivité était socialement excusée par cet abus. Tout comme elles, leur mère vivait dans un contexte de violence.

La psychothérapie intervient souvent dans un moment de crise durant lequel la femme se sent impuissante. L'espace thérapeutique offre la possibilité de (re)construire un espace de sécurité pour travailler sur soi. C'est le moment pour elle de comprendre la séquence et la logique des événements, leurs implications dans les diverses sphères de sa vie et de celles de ses proches et le sens pris par la violence dans son histoire personnelle. C'est aussi

Alterstice - Revue Internationale de la Recherche Interculturelle, vol. 3, $n^{\circ} 2$ 
le moment d'expérimenter, par le biais de la relation thérapeutique, un autre mode de relation, sans menaces et sans assujettissement. À partir de cette expérience psychothérapeutique, il est possible que la personne retrouve les éléments perdus et anéantis par la violence et restaure ses capacités créatrices.

Au Brésil, selon Narvaz et Koller (2006) et Bifano (2002), le désir d'avoir une famille et de la maintenir unie, la pression sociale sur le modèle familial traditionnel, la peur, l'isolement social et les risques de perdre son identité " soignante/protectrice » sont quelques-uns des facteurs qui contribuent à ce que la femme se maintienne dans des relations de violence et conserve le statu quo. Étant donné la représentation des fonctions sociales masculines et féminines, normalisées également par les influences religieuses en Amérique Latine (Martin-Baró, 1972), sortir d'une situation de violence conjugale semble souvent une tâche insurmontable, voire impensable. Rompre avec la famille peut être vécu comme une trahison envers les proches et envers soi-même, car cela va à l'encontre du rôle réservé socialement, ce rôle étant intimement lié à la fonction maternelle et à celle de " soignante/protectrice " (Rocha-Coutinho, 1994).

Les caractéristiques et les fonctions féminines sont souvent liées, dans la culture brésilienne, à des représentations valorisant la docilité, l'acceptation et l'abnégation (Rocha-Coutinho, 1994; Santi, Nakano et Lettiere, 2010). Dans le cas des femmes victimes de violence conjugale, à cette représentation s'ajoute l'existence d'une déchirure dans le tissu social, qui restreint l'existence de la femme à celle de son conjoint : à ses côtés, elle croit avoir une fonction privilégiée, être la seule personne à le connaître, le comprendre et pouvoir l'aider. Aux prises avec des difficultés à trouver sa véritable identité et un autre rôle, c'est dans la relation violente qu'elle se reconnaît. Les voies prises par ces femmes victimes pour exprimer leur plainte ne leur offrent ainsi que très rarement la réponse qu'elles attendaient. La séparation, le divorce et l'emprisonnement du conjoint ne sont en effet pas des issues qu'elles avaient envisagées et, pour plusieurs, ces possibilités ne font partie ni des solutions culturellement acceptées, ni même des solutions envisageables en général. En ce sens, le défi pour la société brésilienne est de refuser toute normalité à la violence, et plus précisément à la violence conjugale, en modifiant les croyances, les valeurs et les pratiques institutionnelles et sociales concernant la représentation du masculin et du féminin (Gomes, 2005; Nobre et Barreira, 2008).

\section{Considérations finales}

II est à noter que les observations présentées ici concernent un effectif limité de femmes brésiliennes se trouvant en situation de violence conjugale. Elles offrent toutefois des similitudes avec le vécu décrit par des femmes d'autres régions du monde exposées à la violence conjugale et elles illustrent la spécificité brésilienne. Au Brésil, on observe en effet une forte influence du réseau social dans la recherche de solutions. Cette influence est cependant à double tranchant: d'une part, le réseau social accompagne la femme et son conjoint dans la recherche d'une solution, ce qui peut avoir un effet moins menaçant, mais, d'autre part, le réseau social, enraciné dans la culture et émanant de celle-ci, peut constituer un obstacle aux changements s'il renforce les conceptions conservatrices et traditionnelles des rôles masculins et féminins.

La judiciarisation constitue une modalité importante de gestion de la faute et du pardon, et aussi de la honte et de I'humiliation, ce qui pourrait contribuer à la reconstruction de l'identité de la personne agressée (Benghozi, 2005). Confrontée à sa propre impuissance à sortir de cette situation, la femme peut décider d'aller plus loin dans l'expression de sa plainte. La violence conjugale devient donc, du fait d'un manque d'accès à d'autres ressources (de santé et de services sociaux), une affaire de justice et de sécurité. Mais, dans ces lieux, les femmes ne se sentent ni entendues, ni protégées. Elles n'arrivent pas à parler de ce dont elles souffrent et de leur subjectivité. Elles ne peuvent pas exprimer les émotions liées à la violence qu'elles ont vécue, pas plus que les peurs suscitées par l'idée - souvent insoutenable - qu'en se séparant elles ne seront plus la femme qu'on leur demande d'être culturellement et que - par leur démarche - leur conjoint sera mis en accusation. Plusieurs extraits de séances illustrent ces observations : on y voit des juges d'instruction s'indignant en voyant la femme hésitante, souffrante, face à la possibilité de voir son conjoint accusé (Bragagnolo, 2012). Deux conceptions différentes s'affrontent alors, s'appuyant chacune sur deux références différentes : l’une sur la justice et l'autre sur la subjectivité. 
Il est donc impératif de réaffirmer l'importance d'un réseau de sécurité publique comme ressource contre la violence conjugale, afin de promouvoir les politiques de protection existantes, par exemple le retrait temporaire de I'agresseur. Le CREMV peut constituer une solution intéressante pour ces femmes, par sa politique d'accueil et par ses objectifs. Cependant, en pratique, on a constaté dans la municipalité étudiée un certain isolement du CREMV par rapport aux services sociaux et de santé, et ce, probablement du fait de son appartenance légale et culturelle au ministère de la Justice et des Droits de l'homme. Cela a pour conséquence d'isoler une fois de plus les femmes. II est essentiel - si l'accueil continue à être réalisé par un organisme relevant de la sécurité - que celui-ci soit bien articulé au réseau de la santé et des services sociaux. La proximité de ces relations institutionnelles permet en effet d'intervenir auprès des familles et des personnes impliquées directement dans des situations de violence conjugale (instigateurs et victimes) au sein de leur communauté d'appartenance et selon les principes de la promotion et de la prévention de la santé, comme cela est prévu dans les politiques publiques du Brésil. Il est également important de mettre en place des mesures sociales qui permettent - effectivement - à la femme d'avoir une aide financière temporaire (pour sa subsistance) ainsi qu'un lieu de vie protégé. Il y a bien sûr les maisons d'hébergement, mais hélas - celles-ci sont en nombre nettement insuffisant. Même s'll est vrai que la violence conjugale n'est pas un phénomène spécifique aux classes sociales défavorisées, il est aussi vrai que, dans ces cas-là, elle est aggravée par la condition d'exclusion sociale déjà engendrée par la pauvreté.

La violence conjugale - qu'elle soit physique, psychologique, verbale ou autre - atteint l'identité, la manière d'être dans le monde, la capacité de donner du sens et la créativité, ce qui empêche la victime de penser et d'envisager une issue à sa situation. Sans une intervention professionnelle et adaptée à son contexte culturel, la femme continuera son errance solitaire. Sans des interventions lui offrant un autre modèle de relations affectives, la femme tendra à répéter le modèle existant, dans une tentative de réparer son histoire après la séparation. Pour cette raison - entre autres -, la violence tend à devenir cyclique et, apparemment, sans issue.

Culturellement, la violence conjugale au Brésil continue d'être perçue d'une manière fragmentée (Santi, Nakano et Lettiere, 2010). Elle est surtout une affaire de famille ou d'État, les deux se révélant impuissants, pour le moment, à changer cet état de choses. La femme en situation de violence conjugale est fréquemment victimisée, infantilisée et considérée comme incapable de prendre en charge sa vie. Faire face à cette situation exige pourtant d'elle qu'elle s'ouvre à la possibilité d'une relation qui ne soit pas basée sur la soumission à la volonté d'un autre. Il est donc important d'explorer toute alternative à cette situation : ressources culturelles, familiales et réseau social sur lesquels la femme pourrait compter et s'appuyer pour se réinventer. II est également essentiel pour elle de comprendre son histoire passée pour être auteure de la suite de son existence et élaborer une nouvelle subjectivité féminine.

\section{Références bibliographiques}

Alves, F. (2006, août). Lei Maria da Penha: das discussões à aprovação de uma proposta concreta de combate à violência doméstica e familiar contra a mulher [en ligne]. Jus Navigandi, 11(1133). http://jus.com.br/revista/texto/8764 (consulté le 15 octobre 2012)

Benghozi, P. (2005). Resiliência familiar e conjugal numa perspectiva psicanalítica dos laços. Psicologia Clínica, 17(2), 101-109.

Bifano, A. (2002). Relacionamentos que Matam: violência conjugal. Universidade Federal de Santa Catarina [en ligne]. Projeto RIZOMA. http://www.rizoma.ufsc.br/pdfs/217-of1-st2.pdf

Bragagnolo, R. (2012). Experiências e lições em uma Vara Criminal e Juizado de Violência Doméstica e Familiar contra a Mulher em Santa Catarina (thèse de doctorat, Universidade Federal de Santa Catarina, Brésil). En ligne à : repositorio.ufsc.br/xmlui/handle/123456789/96177

Campbell, J.,Webster, D. et Glass, N. (2009). The Danger Assessment: validation of a lethality risk assessment instrument for intimate partner femicide. Journal of Interpersonal Violence, 24(4), 653-674.

Gomes, I. (2005). Transmissão psíquica transgeracional e violência conjugal: um relato de caso. Boletim de Psicologia, 55(123), 177-188.

Alterstice-Revue Internationale de la Recherche Interculturelle, vol. 3, $n^{\circ} 2$ 
Izumino, W. (2003). Justiça para todos: Os juizados especiais criminais e a violência de gênero (thèse de doctorat, Universidade de São Paulo, Brésil). En ligne à :

http://www.nevusp.org/portugues/index.php?option=com_content\&task=view\&id=795\&ltemid=96

Mariz, N. (2008). Conjugalidade e violência: uma escuta em terapia familiar psicanalítica. Anais do III Congresso Internacional de Psicopatologia Fundamental, Niterói. En ligne à :

http://www.psicopatologiafundamental.org/uploads/files/iii_congresso/mesas_redondas/conjugalidade_e_ violencia.pdf

Martín-Baró, I. (1972). Psicodiagnóstico de América Latina. San Salvador : Editorail LEA.

Martins-Borges, L. et Leveillée, S. (2005). L’homicide conjugal commis au Québec: observations préliminaires des différences selon le sexe des agresseurs. Pratiques Psychologiques, 11, 47-54.

Narvaz, M. et Koller, S. (2006). Mulheres vítimas de violência doméstica: compreendendo subjetividades assujeitadas. Revista Psico, 37(1), 7-13.

Nobre, M. et Barreira, C. (2008). Controle social e mediação de conflitos: as delegacias da mulher e a violência doméstica. Sociologias, 20, 138-163.

Pocreau, J.-B. et Martins Borges, L. (2013). La cothérapie en psychologie clinique interculturelle. Santé Mentale au Québec, 38(1), 227-242.

Prefeitura Municipal de Florianópolis. (2012). Centro de Referência de Atendimento à Mulher em Situação de Violência-PAEFI. Secretaria Municipal de Assistência Social [en ligne].

http://www.pmf.sc.gov.br/entidades/semas/index.php?pagina=servpagina\&menu=3\&id=4610 (consulté le 20 octobre 2012)

Rocha-Coutinho, M. (1994). Tecendo por trás dos panos. A mulher brasileira nas relações familiares. Rio de Janeiro (Brésil) : Rocco.

Santi, L., Nakano, A. et Lettiere, A. (2010). Percepção de mulheres em situação de violência sobre o suporte e apoio recebido em seu contexto social. Texto \& Contexto - Enfermagem, 19(3), 417-424.

Secretaria de Políticas para as Mulheres. (2012). Violência Doméstica no Brasil - Relatório Anual 2011 da Central de Atendimento à Mulher - Disque 180 [en ligne]. http://www.sepm.gov.br/noticias/ultimas_noticias/07fevereiro-relatorio-180 (consulté le 24 septembre 2012)

Supremo Tribunal Federal. (2012, 6 février). Supremo julga procedente ação da PGR sobre Lei Maria da Penha [en ligne]. http://www.stf.jus.br/portal/cms/verNoticiaDetalhe.asp?idConteudo=199853 (consulté le 20 octobre 2012)

Waiselfisz, J. (2012, août). Mapa da Violência. 2012. Atualização: homicídio de mulheres no Brasil. En ligne à : http://www.mapadaviolencia.org.br/pdf2012/MapaViolencia2012_atual_mulheres.pdf

Wilson, M. et Daly, M. (1993). Spousal homicide risk and estrangement. Violence and Victims, 1, 3-16. 